\title{
The Neurofibromatosis 1 Gene Product Neurofibromin Regulates Pituitary Adenylate Cyclase-Activating Polypeptide-Mediated Signaling in Astrocytes
}

\author{
Biplab Dasgupta, Laura L. Dugan, and David H. Gutmann \\ Department of Neurology, Washington University School of Medicine, St. Louis, Missouri 63110
}

\begin{abstract}
Individuals with the neurofibromatosis 1 (NF1)-inherited tumor predisposition syndrome develop low-grade astrocytomas. The NF1 tumor suppressor gene product neurofibromin exhibits GTPase-activating activity (GAP) toward RAS, such that loss of neurofibromin expression leads to high levels of activated RAS and increased cell proliferation. Previous work has demonstrated that Nfl inactivation in astrocytes leads to increased cell proliferation in vitro and in vivo, accompanied by increased RAS pathway activation. Studies on NfI mutant Drosophila have suggested that neurofibromin might also regulate cAMP signaling. Because intracellular cAMP levels have profound effects on astrocyte growth control, we sought to determine the contribution of neurofibromin to astrocyte cAMP regulation. In this report, we demonstrate that $N f 1$ inactivation in astrocytes results in reduced cAMP generation in response to PACAP and attenuated calcium influx and Rap1 activation. Based on the differential effects of forskolin and dibutyryl-cAMP on Nf1-I- astrocytes, neurofibromin likely functions at the level of adenylyl cyclase activation. Last, the reintroduction of a fragment of neurofibromin containing residues sufficient for restoring RAS-GAP function in $\mathrm{Nf1}-1$ - cells resulted in only partial restoration of neurofibromin-mediated cAMP regulation. These results demonstrate that neurofibromin positively influences cAMP generation and activation of cAMP growth regulatory targets in astrocytes and expands the role of the NF1 gene in astrocyte growth regulation.
\end{abstract}

Key words: cAMP; glia; RAS; GAP; Rap1; astrocyte

\section{Introduction}

Neurofibromatosis 1 (NF1) is an inherited tumor predisposition syndrome in which affected individuals are prone to the development of optic nerve glioma, composed of neoplastic astrocytes (Listernick et al., 1999). NF1-associated optic nerve gliomas (astrocytomas) exhibit biallelic inactivation of the NF1 tumor suppressor gene and loss of neurofibromin expression (Gutmann et al., 2000; Kluwe et al., 2001). Based on the presence of a 300 amino acid residue RAS GTPase-activating protein (GAP)related domain (NF1GRD), neurofibromin growth regulation has been hypothesized to reflect its ability to function as a RASGAP (Ballester et al., 1990; Martin et al., 1990; Xu et al., 1990). In this regard, loss of neurofibromin in NF1-associated tumors is associated with increased RAS activity, which can be ameliorated by re-expression of wild-type NF1GRD (Basu et al., 1992; DeClue et al., 1992; Bollag et al., 1996; Hiatt et al., 2001).

Although it is tempting to attribute all of neurofibromin growth regulatory function to RAS regulation, the NF1GRD represents only $10 \%$ of the entire molecule. Studies in Drosophila have suggested that neurofibromin also has non-RAS-GAP func-

\footnotetext{
Received June 24, 2003; revised Aug. 11, 2003; accepted Aug. 13, 2003.

This work was supported by National Institutes of Health Grant NS36996 and United States Army Grant DAMD1703-1-0215 (D.H.G.). We thank Drs. D. Wade Clapp and Feng-Chun Yang (Indiana University) for MSCV Nf1GRD Pac and MSCV-Pac virus and Dr. Akira Arimura (Tulane University) for PAC-1-R antibodies.

Correspondence should be addressed to Dr. David H. Gutmann, Department of Neurology, Box 8111, 660 South Euclid Avenue, St. Louis, M0 63110. E-mail: gutmannd@neuro.wustl.edu.

Copyright $\odot 2003$ Society for Neuroscience $\quad$ 0270-6474/03/238949-06\$15.00/0
}

tions that involve regulation of intracellular cAMP (Guo et al., 1997). In these studies, Drosophila Nf1 was required for activation of adenylyl cyclase by the neuropeptide, PACAP (pituitary adenylate cyclase activating peptide), which potently increases cAMP levels in pituitary cells (for review, see Waschek, 2002). Moreover, the small size defect of Nf1-deficient Drosophila was rescued by overexpression of activated protein kinase A (PKA), but not by manipulating RAS signaling (The et al., 1997). The rescue by PKA and not RAS supports the notion that Drosophila neurofibromin regulates CAMP signaling and that some of the phenotypes in Drosophila that result from Nf1 inactivation are RASindependent. Similarly, neurofibromin also regulates G-proteinstimulated adenylyl cyclase activity in mammalian neurons (Tong et al., 2002).

In astrocytes, we have previously shown that increased intracellular cAMP provides a strong growth inhibitory signal, and increased intracellular cAMP leads to activation of Rap1, which in turn, inhibits Raf-1-mediated RAS signaling (Dugan et al., 1999; Apicelli et al., 2003). If neurofibromin is required for propagation of astrocyte PACAP-CAMP signaling, we predict that Nf1-deficient astrocytes would exhibit impaired cAMP signaling in response to PACAP, leading to increased cell proliferation. In this report, we demonstrate that $N f 1$-deficient astrocytes have impaired cAMP responses to PACAP, associated with decreased calcium influx and Rap1 activation, and that neurofibromin functions at the level of adenylyl cyclase activation. Last, using a neurofibromin fragment containing residues sufficient to restore 
RAS-GAP activity in $\mathrm{Nf1}-/$ - cells, we observed only a partial restoration of Nf1-mediated cAMP regulation. These results suggest that one component of neurofibromin growth regulation involves RAS-independent, cAMP-mediated, signaling, and that residues outside of the RAS-GAP domain might be responsible for this effect.

\section{Materials and Methods}

Primary astrocyte culture and establishment of $\mathrm{Nf1-/}$ - astroglial cultures. Murine neocortical astroglial cultures, containing $>95 \%$ of GFAPimmunoreactive cells (astrocytes), were generated from postnatal day 2

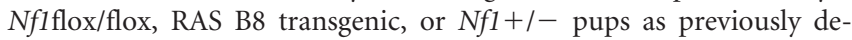
scribed (Gutmann et al., 2001). To eliminate neurofibromin expression, Nf1flox/flox astrocytes were treated with Ad5-Cre (University of Iowa Gene Transfer Vector core, Iowa City, IA) or Ad5-lacZ (control) for $2 \mathrm{hr}$ at a multiplicity of infection of 1:50, washed twice, and maintained for at least $4 \mathrm{~d}$. Loss of neurofibromin was confirmed by Western blot. All experiments were performed on passage 1 (P1) or P2 cultures. Mice were used in accordance with established Animal Studies Protocols at the Washington University School of Medicine. Genotypes were determined by PCR from tail DNA.

Measurement of intracellular cAMP. Intracellular cAMP was measured using the cAMP Enzyme Immunoassay kit (Cayman Chemical, Ann Arbor, MI) according to manufacturer's instructions. Briefly, equal numbers of $\mathrm{Nf1}+\mathrm{I}+, \mathrm{Nf1}-\mathrm{I}$ - or $\mathrm{Nf1}+\mathrm{I}$ - astrocytes were serum-starved for $48 \mathrm{hr}$ and stimulated with either medium only (control), PACAP $\left(10^{-13}\right.$, $10^{-11}$, or $\left.10^{-9} \mathrm{M}\right)$, EGF $\left(1.6 \times 10^{-9} \mathrm{M}\right)$, or forskolin $(10 \mu \mathrm{M})$ for $10 \mathrm{~min}$ at $37^{\circ} \mathrm{C}$. For the RAS inhibition experiments, astrocytes were serumstarved for $48 \mathrm{hr}$ in presence of either L-744,832 (20 $\mu \mathrm{M})$ or vehicle (DMSO). The concentrations of PACAP, forskolin, and L-744,832 were chosen based on previous studies in astrocytes (Tatsuno et al., 1996; Dugan et al., 1999; Feldkamp et al., 1999). Astrocytes were washed three times with ice-cold PBS, lysed in chilled ethanol, centrifuged at $3.5 \mathrm{~K}$ for $15 \mathrm{~min}$ at $4^{\circ} \mathrm{C}$, and resuspended in $200 \mu \mathrm{l}$ of assay buffer after SpeedVac evaporation of the supernatant. All experiments were performed in triplicate or quadruplicate, and each experiment was repeated three times with identical results. Protein concentration was determined from the ethanol-insoluble pellet using the BCA reagent (Pierce, Rockford, IL).

Rapl activity assay. Rap1 activity was assayed as previously described (Apicelli et al., 2003). Briefly, equal numbers of $\mathrm{Nf1}+/+$ and $\mathrm{Nf1}-\mathrm{I}-$ astrocytes were serum-starved for $48 \mathrm{hr}$ and incubated with either medium alone (control), PACAP $\left(10^{-11}\right.$ and $\left.10^{-9} \mathrm{M}\right)$, or dibutyryl-cAMP (db-cAMP) $(300 \mu \mathrm{M})$ for $10 \mathrm{~min}$ at $37^{\circ} \mathrm{C}$. Cells were washed with cold PBS, lysed in cold MAPK (mitogen-activated protein kinase) buffer, cleared by centrifugation, and incubated with glutathione beads containing $30 \mu \mathrm{g}$ of glutathione $S$-transferase (GST)-tagged RalGDS (a gift from J. L. Bos, University Medical Center, Utrecht, The Netherlands) at $4^{\circ} \mathrm{C}$ for $1 \mathrm{hr}$. The beads were washed four times in cold MAPK buffer, and bound proteins were eluted by boiling in $2 \times$ Laemmli buffer, followed by SDS-PAGE separation and Western blotting. Each experiment was performed at least three times with identical results.

Western blot analysis. Western blots were performed as previously described (Apicelli et al., 2003) after transfer to polyvinylidene difluoride membranes (Immobilon; Fisher Scientific, Dallas, TX). Neurofibromin and the NF1GRD were detected using anti-NF1GRP-D (sc67, 1:300 dilution; Santa Cruz Biotechnology, Santa Cruz, CA) and anti-KT3 antibodies (MMS-125P, 1:1000 dilution; Babco, Richmond, CA), respectively. Other antibodies used include Rap1 (sc65, 1:500 dilution; Santa Cruz Biotechnology), MAPK and phospho-MAPK (9102 and 9106, 1:1000 dilution; Cell Signaling Technology, Beverly, MA), and PAC-1-R (1:1000 dilution; provided by Dr. Akira Arimura, Tulane University, New Orleans, LA). Appropriate HRP-conjugated secondary rabbit or mouse antibodies (Sigma, St. Louis, MO) were used for detection by enhanced chemiluminescence (Amersham, Piscataway, NJ).

Calcium influx assay. Calcium uptake assay was performed as previously described (Dugan et al., 1996) with minor modifications. Equal numbers of astrocytes were grown in $35 \mathrm{~mm}$ dishes, serum-starved for 48 $\mathrm{hr}$, and incubated with medium only (control), PACAP $\left(10^{-11} \mathrm{M}\right)$, or
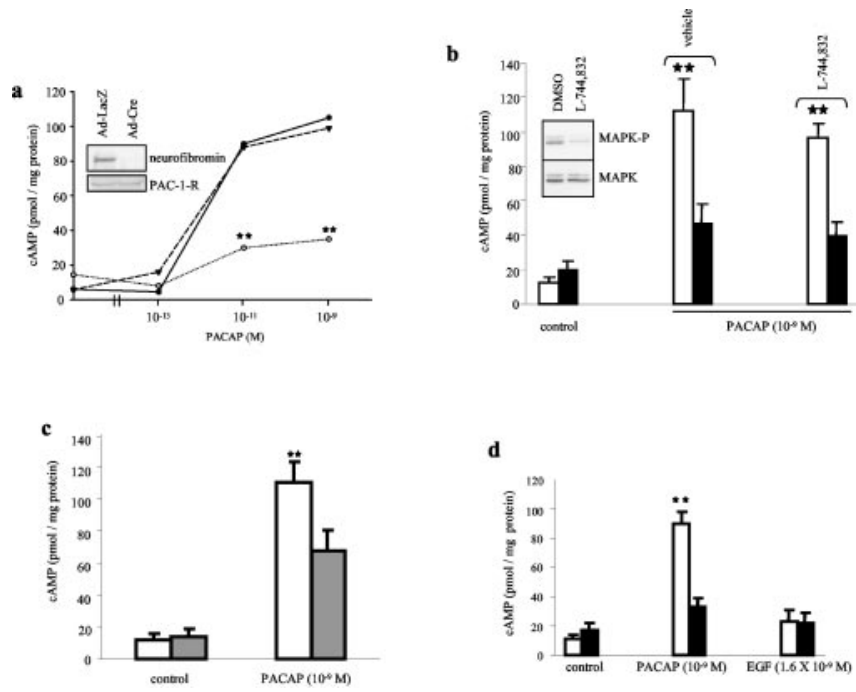

Figure 1. PACAP-induced CAMP generation is impaired in both $N f 1-/-$ and $N f 1+/-$ astrocytes and is independent of RAS activation. $a$, Nf1 - / - astrocytes exhibit a marked decrease in CAMP generation in response to various concentrations of PACAP. CAMP produced by $\mathrm{Nf1}+\mathrm{I}+$ (solid line), Nf1-I- (dotted), and RAS transgenic B8 astrocytes (dashed) are shown. The inset illustrates neurofibromin and PAC-1-R expression in $\mathrm{Nf1}+\mathrm{I}+$ and $\mathrm{Nf1}-\mathrm{I}-$ astrocytes after adenoviral transduction. $b$, Pretreatment of either Nf1+/+ (white bar) or Nf1 - / - astrocytes (black bar) with the RAS farnesyltransferase inhibitor $(L-744,832)$ did not significantly alter PACAP-mediated CAMP generation in Nf1-/ - or wild-type astrocytes. The inset demonstrates that L-744,832 treatment resulted in inhibition of MAPK activity (phosphoMAPK) without altering total MAPK (MAPK) expression. C, CAMP generation in response to PACAP stimulation in also impaired in $\mathrm{Nf1}+1-$ astrocytes (gray bar) compared with $\mathrm{Nf1}+\mathrm{I}+$ (white bar) astrocytes. $d$, Whereas differences in cAMP levels were observed in Nf1 + / + (white bar) versus Nf1-1- (black bar) astrocytes in response to PACAP treatment, no differences were observed in response to EGF treatment. Each error bar represents mean $\pm \mathrm{SD}$. Asterisks denote statistically significant differences $(p<0.004)$.

with db-cAMP (300 $\mu \mathrm{M}$; Dugan et al., 1999) in the presence of $45 \mathrm{Ca}^{2+}(4$ $\mu \mathrm{Ci} /$ culture dish; Perkin-Elmer, Boston, MA) at $37^{\circ} \mathrm{C}$ for $30 \mathrm{~min}$. Cells were washed three times with cold PBS, lysed in $500 \mu \mathrm{l}$ of $0.2 \%$ SDS for 10 $\min$, and $45 \mathrm{Ca}^{2+}$ incorporation was determined by scintillation counting. Parallel plates of $\mathrm{Nf1}+/+$ and $\mathrm{Nf1}-/$ - astrocytes were used for cell counting and protein determination to demonstrate equal number of astrocytes per condition. Each experiment was performed at least three times with identical results.

Retroviral transduction of astrocytes. Retroviral transduction was performed as previously reported (Hiatt et al., 2001) with minor modifications. $N$ flflox/flox astrocyte cultures containing equal numbers of astrocytes were infected twice, first for $4 \mathrm{hr}$ and then overnight with either murine stem cell virus (MSCV)-Pac or MSCV-NF1GRD-Pac in the presence of $10 \mu \mathrm{g} / \mathrm{ml}$ polybrene (Specialty Media, Phillipsburg, NJ). Fortyeight hours after infection, transduced astrocytes were selected in medium containing $2 \mu \mathrm{g} / \mathrm{ml}$ puromycin for an additional $48 \mathrm{hr}$. After expression of NF1GRD was confirmed by Western blot, the same plates were used for adenoviral infection with either Ad5-Cre to inactivate Nf1 or Ad5-LacZ (control).

\section{Results}

\section{Neurofibromin is required for PACAP-induced cAMP generation}

To determine whether neurofibromin is required for PACAPmediated astrocyte cAMP production, we disrupted neurofibromin expression in $\mathrm{Nflflox} /$ flox neonatal cortical astrocytes using Cre recombinase (Fig. $1 a$, inset). No changes in PACAP receptor (PAC-1-R) expression were observed at the RNA (data not shown) or protein level (Fig. $1 a$, inset) after $N f 1$ gene inactivation. Serum-starved $N f 1+/+$ and $N f 1-/-$ astrocytes were stimulated with PACAP at several concentrations, and intracellular 
cAMP levels were measured. Intracellular cAMP levels were significantly decreased in $\mathrm{Nf1}-/$ - compared with $\mathrm{Nf1}+/+$ astrocytes in response to both $10^{-9}$ and $10^{-11} \mathrm{M}$ PACAP $(66 \%$ reduction; $p=0.001$ ) (Fig. 1a). Because neurofibromin functions as a GAP for RAS, we next determined whether activated Ha-RAS expression resulted in decreased cAMP levels in response to PACAP. In contrast to Nf1-/ - cells, we observed no effect of activated RAS expression on baseline or PACAP-induced cAMP generation (B8 RAS transgenic astrocytes) (Fig. 1a). In addition, RAS inhibition using the farnesyltransferase inhibitor L-744,832 did not significantly alter cAMP generation in either wild-type or Nf1 - / - astrocytes (14.1 vs $15.6 \%$ reduction, respectively) (Fig. $1 b)$. At this concentration, RAS pathway activation is significantly inhibited, as reflected in reduced activated phosphoMAPK (Fig. $1 b$, inset). Collectively, these results suggest that neurofibromin-regulated cAMP activity is independent of RAS activation.

To demonstrate a direct relationship between neurofibromin expression and cAMP regulation, we examined cAMP generation in $\mathrm{Nf1}+/$ - astrocytes, with $50 \%$ wild-type neurofibromin expression (Bajenaru et al., 2001). We found reduced PACAPinduced cAMP production in $\mathrm{Nf1}+/$ - astrocytes compared with $N f 1+/+$ astrocytes $(38.4 \%$ reduction; $p=0.004)$, supporting a dose-dependent effect of neurofibromin expression on cAMP generation (Fig. 1c).

Because PACAP functions through PAC-1 and PAC-2 G-protein-coupled receptors, we examined neurofibrominmediated cAMP regulation in response to epidermal growth factor (EGF). In contrast to PACAP stimulation, at doses sufficient to activate RAS $\left(1.6 \times 10^{-9} \mathrm{M} \mathrm{EGF}\right.$; data not shown), we observed no effect of EGF on cAMP production in either $\mathrm{Nf1}+/+$ or $N f 1-/-$ astrocytes (Fig. $1 d$ ).

\section{Neurofibromin loss leads to decreased calcium influx and impaired Rap1 activation in response to PACAP} cAMP generation in many cell types results in enhanced calcium influx (Kamp and Hell, 2000) and activation of the small GTPase molecule Rap1 (Schmitt and Stork, 2002; Rangarajan et al., 2003). To determine whether neurofibromin loss impaired downstream effects of PACAP-stimulated cAMP production, we measured calcium influx and Rapl activation in $\mathrm{Nf1-} / \mathrm{-}$ astrocytes in response to PACAP stimulation $\left(10^{-9}\right.$ and $\left.10^{-11} \mathrm{M}\right)$. We observed increased Rap1 activation at both PACAP concentrations in $\mathrm{Nf1}+\mathrm{I}+$ astrocytes, using the GST-RalGDS pulldown assay. In contrast, PACAP-stimulated Rap 1 activation was significantly reduced in $N f 1-/-$ astrocytes ( $p=0.001$ ) (Fig. 2a). Scanning densitometric values for Rap1-GTP in the $N f 1+/+$ astrocytes were 17.66 and 15.5 after normalization to total Rap1, respectively, in response to $10^{-9}$ and $10^{-11} \mathrm{M}$ PACAP. The corresponding values for the $\mathrm{Nf1-/-}$ astrocytes were 2.8 and 1.98 after normalization. Similarly, we observed a significant reduction in calcium influx in $\mathrm{Nf1}-/-$ astrocytes compared with $\mathrm{Nf1}+/+$ astrocytes in response to PACAP $\left(10^{-9}\right.$ and $\left.10^{-11} \mathrm{M}\right)$ stimulation ( $p=0.001$ ) (Fig. $2 b$ ). Collectively, these results demonstrate that neurofibromin loss not only affects cAMP generation, but also impairs downstream signaling mediated by cAMP in astrocytes.

Neurofibromin mediates PACAP-induced cAMP generation at the level of adenylyl cyclase

To determine the level at which neurofibromin regulates PACAP-induced CAMP generation, we treated cells with forskolin $(10 \mu \mathrm{M})$ to activate adenylyl cyclase or the cAMP analog db-
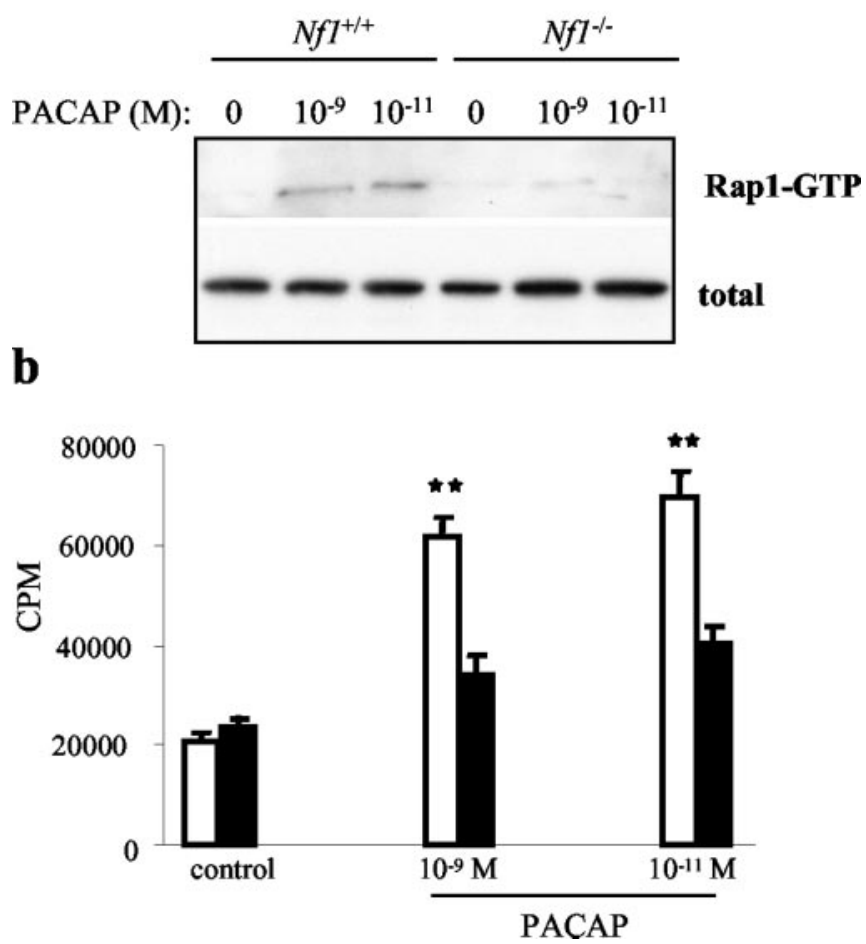

Figure 2. PACAP activation of Rap1 and induction of calcium influx is impaired in Nf1-/astrocytes. a, Serum-starved Nf1 + I+ and Nf1-/ - astrocytes stimulated with PACAP were analyzed for Rap1 activation. The top panel denotes active Rap1 (Rap1-GTP bound to GSTRalGDS), whereas bottom panel demonstrates the total Rap1 in each sample. Significantly reduced Rap1 activation is observed in the Nf1 - I- compared with Nf1 / I+ astrocytes. b, Serum-starved $\mathrm{Nf1}+1+$ and $N f 1-/-$ astrocytes stimulated with PACAP in presence of 45 $\mathrm{Ca}^{2+}$ demonstrate decreased calcium influx in Nf1 - / - (black bar) compared with Nf1 + I+ (white bar) astrocytes. Each error bar represents mean \pm SD. Asterisks denote statistically significant differences $(p=0.001)$.

cAMP. We observed a reduction in forskolin-stimulated cAMP generation in $\mathrm{Nf1}-/ \mathrm{-}$ compared with $\mathrm{Nf1}+/+$ astrocytes $(p=$ 0.007 ) (Fig. $3 a$ ). In contrast, $N f 1+/+$ and $N f 1-/-$ astrocytes treated with $300 \mu \mathrm{M}$ db-cAMP exhibited similar elevations in intracellular calcium $(p=0.001)$ (Fig. 3b). Similarly, we observed nearly identical levels of Rap 1 activation in Nf1-/ - compared with $\mathrm{Nf1}+/+$ astrocytes in response to db-cAMP treatment (Fig. 3c). By scanning densitometry, the levels of active Rap1, after normalization to total Rap1, were identical in $N f 1-/-$ and $N f 1+/+$ astrocytes (19.66 and 20.02 for $N f 1+/+$ and $N f 1-/-$ astrocytes, respectively). These results demonstrate that neurofibromin functions at the level of adenylyl cyclase activation.

Ectopic expression of the NF1-GAP-related domain partially rescues the cAMP response to PACAP in Nf1-/ - astrocytes To determine whether a neurofibromin fragment sufficient to restore neurofibromin RAS-GAP function (Hiatt et al., 2001) could also restore neurofibromin-mediated cAMP responses to PACAP, we transduced Nf1 flox/flox astrocytes with MSCV containing either empty vector (MSCV-Pac) or the human NF1GRD fragment (MSCV-NF1GRD-Pac) before $N f 1$ gene inactivation by Ad5-Cre. The NF1GRD was tagged with the KT3 epitope to facilitate detection (Fig. 4, inset), but was also detected using neurofibromin-specific antibodies (data not shown). After PACAP $\left(10^{-9} \mathrm{M}\right)$ stimulation, cAMP levels increased signifi- 
$\mathbf{a}$
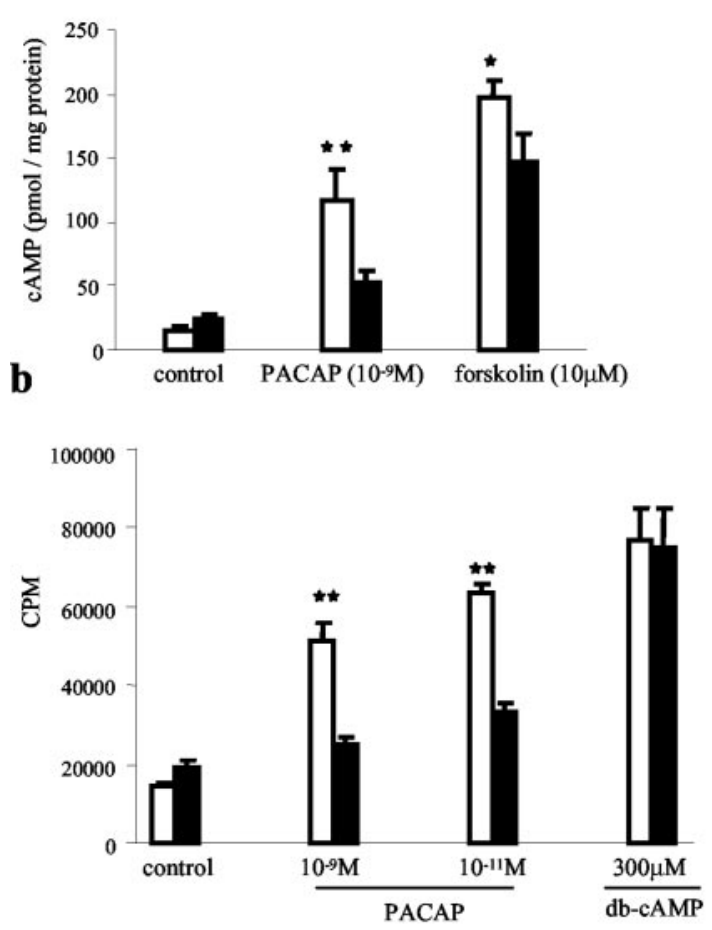

c

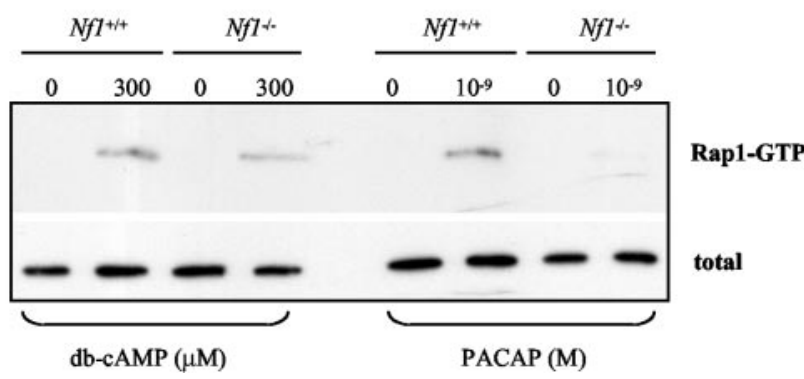

Figure 3. Forskolin-stimulated CAMP generation is reduced in Nf1-/- astrocytes, whereas $\mathrm{db}$-CAMP-induced calcium influx and Rap1 activation is similar in both Nf1-I- and $\mathrm{Nf1}+1+$ astrocytes. $a$, cAMP generation in response to PACAP and forskolin is reduced in Nf1 - I - astrocytes (black bars) compared with $N f 1+1+$ astrocytes (white bar). b, Calcium influx in response to PACAP is reduced in Nf1- - astrocytes (black bars) compared with $\mathrm{Nf1}+/+$ astrocytes (white bar), but equivalent in response to db-CAMP stimulation. $\mathrm{C}$, Whereas Rap1 activation (Rap1-GTP) in response to PACAP is reduced in Nf1-/ - compared with $N f 1+/+$ astrocytes, equivalent levels of Rap1 activation were observed in response to db-cAMP. Each error bar represents mean \pm SD. Asterisks denote statistically significant differences $\left({ }^{*} p=0.001 ;{ }^{* *} p=0.007\right)$.

cantly in Nf1-/- astrocytes transduced with MSCV-NF1GRD$\mathrm{Pac}(p=0.005)$, compared with $N f 1-/-$ astrocytes transduced with MSCV-Pac vector control (Fig. 4). The cAMP increase in $\mathrm{Nf1}-\mathrm{I}-$ astrocytes expressing NF1GRD was intermediate between the increase observed in $\mathrm{Nf1}+/+$ and $\mathrm{Nf1-/}-$ astrocytes in response to PACAP. The differential cAMP response to PACAP between $N f 1+/+$ and $N f 1-/-$ astrocytes was found to be unaffected by retroviral infection, because there were no differences in cAMP production between uninfected $\mathrm{Nf1}-/$ - astrocytes and $\mathrm{Nf1}-\mathrm{I}-$ astrocytes infected with MSCV-Pac or uninfected $N f 1+/+$ astrocytes and those infected with either MSCVPac or MSCV-NF1GRD-Pac. Although we observed a partial restoration of cAMP generation in $N f 1-/-$ astrocytes expressing NF1GRD, these results suggest that NF1GRD expression at levels sufficient to completely restore RAS-GAP function are not sufficient to restore neurofibromin cAMP regulation.

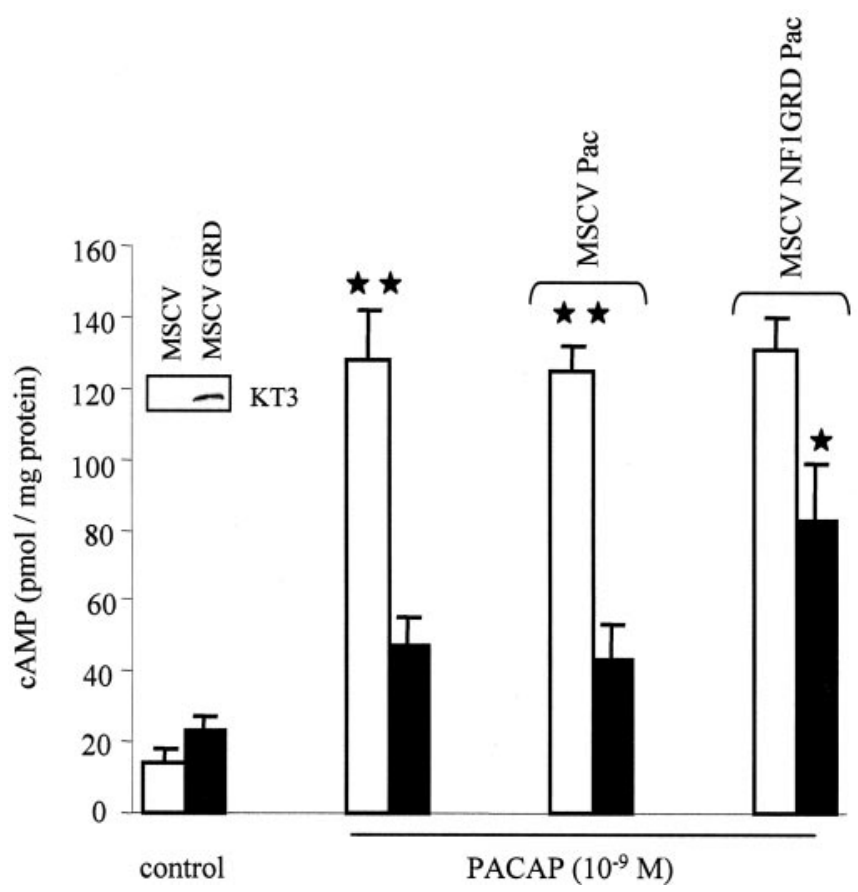

Figure 4. Ectopic expression of NF1GRD partially rescues the impaired CAMP response to PACAP in Nf1 - I- astrocytes. Whereas reduced cAMP levels were observed in untransduced and vector transduced $N f 1-/$ - (black bars) compared with Nf1 + I+ (white bars) astrocytes, a partial restoration of this response was observed in astrocytes expressing the NF1GRD fragment. No effect on CAMP generation was observed in Nf1+/+ astrocytes expressing the NF1GRD fragment. Expression of KT3-tagged NF1GRD in Nf1 - / - MSCV-NF1GRD-Pac treated astrocytes is shown in the inset. Each error bar represents mean \pm SD. Asterisks denote statistically significant differences $\left({ }^{*} p=0.001 ;{ }^{* *} p=0.005\right)$.

\section{Discussion}

Previous studies on astrocyte mitogenic signaling in NF1 have focused on the role of neurofibromin in regulating RAS activity. In both human astrocytoma and Nf1-deficient mouse astrocytes, elevated levels of RAS and RAS effector (Akt and MAPK) activity are associated with neurofibromin loss (Lau et al., 2000; Bajenaru et al., 2002), suggesting that neurofibromin RAS regulation is a critical component of its anti-mitogenic and tumor suppressor function. However, recent studies in Drosophila have shown that neurofibromin loss results in attenuation of cAMP generation in response to the PACAP neuropeptide, which can be rescued using an activated PKA allele (Guo et al., 1997; The et al., 1997). Genetic complementation studies in flies have confirmed this defect by positioning $N f 1$ in the amnestic (PACAP receptor) pathway (Yager et al., 2001).

Several findings suggest that non-RAS functions of neurofibromin might have important implications for $\mathrm{Nf1}$-mediated astrocyte growth regulation. First, PACAP and its receptor, PAC-1, are expressed in differentiating cells of the mouse neural tube (Waschek et al., 1998), where they have multiple actions during nervous system development and differentiation (Waschek, 2002). In E10.5 hindbrain neuroepithelial cells, PACAP potently increased cAMP levels and downregulated expression of the PKA-target gene, gli-1, concomitant with a decrease in DNA synthesis. Similarly, in neural stem cells, PACAP induces astrocyte differentiation from precursor cells (Vallejo and Vallejo, 2002). Second, increased intracellular cAMP results in activation of Rap1, an anti-mitogenic RAS pathway antagonist. In the absence of B-raf, which we have previously shown is not expressed at significant levels in neocortical astrocytes, Rap1 activation leads 
to inhibition of Raf-1 activation (Dugan et al., 1999). In this regard, the expression of an activated Rap1 allele in transgenic mouse astrocytes results in decreased astrocyte numbers in vivo and decreased astrocyte proliferation in vitro, associated with reduced MAPK activation (Apicelli et al., 2003). Moreover, the expression of an activated Rap1 allele in astrocyte-restricted RAS transgenic mice results in a marked attenuation of astrocyte proliferation and tumor formation (Apicelli et al., 2003) (E. J. Uhlmann and D. H. Gutmann, unpublished observations).

In this report, we demonstrate a dose-dependent impairment in cAMP generation in $N f 1$ mutant astrocytes, with $N f 1+/-$ and $\mathrm{Nf1}-$ / - exhibiting graded reductions in intracellular cAMP levels in response to PACAP. Our results are in agreement with similar trends observed by others in mouse brain (Tong et al., 2002). We show that downstream pathways activated by cAMP in astrocytes are likewise impaired by neurofibromin loss, including intracellular calcium influx and Rap1 activation. This impairment in CAMP regulation is specific to PACAP and is not observed in response to EGF treatment or RAS activation, suggesting that specific signals are required to activate this pathway and unmask the neurofibromin-deficient phenotype in astrocytes. Interestingly, the baseline cAMP levels in Nf1-/- astrocytes were found to be 1.5- to 1.8-fold higher than that observed in $\mathrm{Nf1}+/+$ astrocytes, as reported for $\mathrm{Nf1}-/-$ Schwann cells (Kim et al., 2001). These baseline differences may result from additional effects of neurofibromin on adenylyl cyclase regulation in quiescent cells that reflect the differential regulation of inhibitory and stimulatory small G-proteins on adenylyl cyclase activation. However, unlike astrocytes, in which cAMP acts as an antimitogenic signal, cAMP is a mitogen for Schwann cells, and neurofibromin functions to antagonize, rather than stimulate, cAMP production (Kim et al., 2001).

We further mapped the level of neurofibromin regulation to the adenylyl cyclase, based on the differential effects of forskolin and db-cAMP stimulation. db-cAMP treatment was equally as effective at inducing intracellular calcium influx and Rap1 activation in $\mathrm{Nf1}+/+$ and $\mathrm{Nf1}-/$ - astrocytes, suggesting that neurofibromin acts upstream of cAMP in the PACAP pathway. In contrast, forskolin activation of adenylyl cyclase resulted in reduced cAMP generation in Nf1-/- astrocytes. Although we observed a reduction in forskolin-mediated cAMP generation, it is not reduced as dramatically as seen after PACAP treatment. Similar trends were observed in Nf1-/- brains (Tong et al., 2002). Collectively, these observations suggest that neurofibromin might function to facilitate adenylyl cyclase activation by PAC-1.

Last, we demonstrate that the re-expression of the NF1-GAPrelated domain in $\mathrm{Nf1}-\mathrm{I}-$ astrocytes can partially restore normal cAMP generation in response to PACAP. In contrast, complete restoration in Drosophila is seen with the full-length neurofibromin (Guo et al., 1997). These results suggest that a portion of neurofibromin-cAMP activity maps to the RAS-GAP domain, but that additional residues might also be important. Because the GAP domain used in these studies (residues 10951569) does not contain the IRA homology domains, it is conceivable that more complete functional restoration will be observed with NF1GRD constructs containing these additional sequences. Experiments are in progress to determine what residues are important for neurofibromin cAMP regulation and to define the mechanism by which neurofibromin modulates PAC-1 activation of adenylyl cyclase. A more complete understanding of this unique function of neurofibromin will provide critical insights into the mechanisms underlying the control of astroglial cell proliferation and differentiation and offer additional targets for therapeutic drug design in patients with NF1-associated astrocytomas.

\section{References}

Apicelli AJ, Uhlmann EJ, Baldwin RL, Ding H, Nagy A, Guha A, Gutmann DH (2003) Role of the Rap1 GTPase in astrocyte growth regulation. Glia 42:225-234.

Bajenaru ML, Donahoe J, Corral T, Reilly KM, Brophy S, Pellicer A, Gutmann DH (2001) Neurofibromatosis 1 (NF1) heterozygosity results in cell autonomous growth advantage for astrocytes. Glia 33:314-323.

Bajenaru ML, Zhu Y, Hedrick NM, Donahoe J, Parada LF, Gutmann DH (2002) Astrocyte-specific inactivation of the neurofibromatosis 1 gene (NF1) is insufficient for astrocytoma formation. Mol Cell Biol 22:5100-5113.

Ballester R, Marchuk D, Boguski M, Saulino A, Letcher R, Wigler M, Collins F (1990) The NF1 locus encodes a protein functionally related to mammalian GAP and Yeast IRA proteins. Cell 63:852-859.

Basu TN, Gutmann DH, Fletcher JA, Glover TW, Collins FS, Downward J (1992) Aberrant regulation of ras proteins in malignant tumor cells from type 1 neurofibromatosis patients. Nature 356:713-715.

Bollag G, Clapp DW, Shih S, Adler F, Zhang YY, Thompson P, Lange BJ, Freedman MH, McCormick F, Jacks T, Shannon K (1996) Loss of NF1 results in activation of Ras signaling pathway and leads to aberrant growth in haematopoietic cells. Nat Genet 12:144-148.

DeClue JE, Papageorge AG, Fletcher JA, Diehl SR, Ratner N, Vass WC, Lowy DR (1992) Abnormal regulation of mammalian p21ras contributes to malignant tumor growth in von Recklinghausen (type 1) neurofibromatosis. Cell 69:265-273.

Dugan LL, Gabrielsen JK, Yu SP, Lin TS, Choi DW (1996) Buckministerfullerenol free radical scavengers reduce excitotoxic and apoptotic death of cultured cortical neurons. Neurobiol Dis 3:129-135.

Dugan LL, Kim JS, Zhang Y, Bart RD, Sun Y, Holtzman DM, Gutmann DH (1999) Differential effects of cAMP in neurons and astrocytes. Role of B-raf. J Biol Chem 274:25842-25848.

Feldkamp MM, Nelson L, Guha A (1999) Growth inhibition of astrocytoma cells by farnesyl transferase inhibitors is mediated by a combination of anti-proliferative, pro-apoptotic and anti-angiogenic effects. Oncogene 18:7514-7526.

Guo HF, The I, Hannan F, Bernards A, Zhong Y (1997) Requirement of Drosophila NF1 for activation of adenylyl cyclase by PACAP-like neuropeptides. Science 276:795-798.

Gutmann DH, Donahoe J, Brown T, James CD, Perry A (2000) Loss of neurofibromatosis 1 (NF1) gene expression in NF1-associated pilocytic astrocytomas. Neuropathol Appl Neurobiol 26:361-367.

Gutmann DH, Wu YL, Hedrick NM, Zhu Y, Guha A, Parada LF (2001) Heterozygosity for neurofibromatosis 1 (NF1) tumor suppressor results in abnormalities in cell attachment, spreading and motility in astrocytes. Hum Mol Genet 10:3009-3016.

Hiatt KK, Ingram DA, Zhang Y, Bollag G, Clapp DW (2001) Neurofibromin GTPase-activating protein-related domains restore normal growth in NF1-/ - cells. J Biol Chem 276:7240-7245.

Lau N, Feldkamp MM, Roncari L, Loehr AH, Shannon P, Gutmann DH, Guha A (2000) Loss of neurofibromin is associated with activation of Ras/MAPK and PI3K/AKT signaling in a neurofibromatosis 1 astrocytoma. J Neuropathol Exp Neurol 59:759-767.

Listernick R, Charrow J, Gutmann DH (1999) Intracranial gliomas in neurofibromatosis 1. Am J Med Genet 89:38-44.

Kamp TJ, Hell JW (2000) Regulation of cardiac L-type calcium channels by protein kinase a and protein kinase C. Circ Res 87:1095-1102.

Kim HA, Ratner N, Roberts TM, Stiles CD (2001) Schwann cell proliferative responses to cAMP and Nf1 are mediated by cyclin D1. J Neurosci 21:1110-1116.

Kluwe L, Hagel C, Tatagiba M, Thomas S, Stavrou D, Ostertag H, von Deimling A, Mautner VF (2001) Loss of NF1 alleles distinguish sporadic from NF1-associated pilocytic astrocytomas. J Neuropathol Exp Neurol 60:917-920.

Martin GA, Viskochil D, Bollag g, McCabe PC, Crosier WJ, Haubruck H, Conroy L, Clark R, O'Connell P, Cawthon RM (1990) The GAP-related domain of the neurofibromatosis type 1 gene product interacts with ras p21. Cell 16:843-849. 
Rangarajan S, Enserink JM, Kuiperij HB, de Rooij J, Price LS, Schwede F, Bos JL (2003) Cyclic AMP induces integrin-mediated cell adhesion through Epac and Rap1 upon stimulation of the beta 2-adrenergic receptor. J Cell Biol 160:487-493.

Schmitt JM, Stork PJ (2002) PKA phosphorylation of Src mediates cAMP's inhibition of cell growth via Rap1. Mol Cell 9:85-94.

Tatsuno I, Morio H, Tanaka T, Hirai A, Tamura Y, Saito Y, Arimura A (1996) Astrocytes are one of the main target cells for pituitary adenylate cyclaseactivating polypeptide in the central nervous system. Ann NY Acad Sci 805:613-619.

The I, Hannigan GE, Cowley GS, Reginald S, Zhong Y, Gusella JF, Hariharan IK, Bernards A (1997) Rescue of Drosophila NF1 mutant phenotype by protein kinase A. Science 276:791-794.

Tong J, Hannan F, Zhu Y, Bernards A, Zhong Y (2002) Neurofibromin regulates G protein- stimulated adenylyl cyclase activity. Nat Neurosci 5:95-96.

Vallejo I, Vallejo M (2002) Pituitary adenylate cyclase-activating polypep- tide induces astrocyte differentiation of precursor cells from developing cerebral cortex. Mol Cell Neurosci 21:671-683.

Waschek JA (2002) Multiple actions of pituitary adenylyl cyclase activating peptide in nervous system development and regeneration. Dev Neurosci 24:14-23.

Waschek JA, Casillas RA, Nguyen TB, DiCicco-Bloom EM, Carpenter EM, Rodriguez WI (1998) Neural tube expression of pituitary adenylate cyclase-activating peptide (PACAP) and receptor: potential role in patterning and neurogenesis. Proc Natl Acad Sci USA 95:9602-9607.

Xu GF, O'Connell P, Viskochil D, Cawthon R, Robertson M, Culver M, Dunn D, Stevens J, Gesteland R, White R (1990) The neurofibromatosis type 1 gene encodes a protein related to GAP. Cell 62:599-608.

Yager J, Richards S, Hekmat-Scafe DS, Hurd DD, Sundaresan V, Caprette DR, Saxton WM, Carlson JR, Stern M (2001) Control of Drosophila perineurial glial growth by interacting neurotransmitter-mediated signaling pathways. Proc Natl Acad Sci USA 98:10445-10450. 